\title{
THE FOURIER-ASYMPTOTIC APPROXIMATION AND ITS APPLICATIONS
}

\author{
MIHAILS BELOVS, JĀNIS SMOTROVS \\ University of Latvia \\ Raiņa bulvāris 19, LV-1586, Rīga, Latvia \\ E-mail: belovs@lanet.lv, smotrovs@lanet.lv
}

\begin{abstract}
The Fourier - asymptotic approximation can be obtained for different types of Fourier series by replacing the Fourier coefficients with their asymptotic $(n \rightarrow+\infty)$ approximations beginning with some index $n$.

We obtain some generalization of the classical Galerkin method for the solution of boundary and spectral problems of ordinary differential equations. The numerical examples show that the addition of asymptotic correction allows us to obtain a high accuracy of results.
\end{abstract}

\section{INTRODUCTION}

The representation of a function in the form of some Fourier series is often used in theoretical constructions. The numerical values of a such function are obtained restricting these Fourier series to the partial sums. Thus, it is supposed that beginning with some index $n$ all Fourier coefficients are annuled.

The main idea of the Fourier-asymptotic (F-A) approximation is that starting with some index $n$ we substitute the Fourier coefficients with their asymptotic $(n \rightarrow+\infty)$ approximations.

Let the function $v=v(x)$ can be expanded in the Fourier series

$$
\begin{gathered}
v(x)=\sum_{k=1}^{+\infty} c_{k} u_{k}(x), \quad x \in(0,1), \quad u_{k}(x)=\sin k \pi x, \\
c_{k}=2 \int_{0}^{1} v(x) u_{k}(x) d x .
\end{gathered}
$$

If we use the partial sum $U=\sum_{k=1}^{n} c_{k} u_{k}$ for the approximation of func- 
tion $v$, then the equality $v=U+r$ hold. The error of the approximation $r=\sum_{k=n+1}^{+\infty} c_{k} u_{k}$ is the remainder of the Fourier series. For example, if the estimate $c_{k}=O\left(1 / k^{2}\right), k \rightarrow+\infty$ hold, then the error satisfy the relation $r=O(1 / n), n \rightarrow+\infty$.

Let we have the asymptotic approximations of Fourier coefficients in the form:

$$
c_{k}=\sum_{\mu=2}^{m} \frac{\alpha_{\mu}}{k^{\mu}}+O\left(\frac{1}{k^{m+1}}\right), \quad k \rightarrow+\infty,
$$

where $m \geq 2$ is the order of asymptotic approximation. Then, if we substitute the Fourier coefficients in the remainder $r=\sum_{k=n+1}^{+\infty} c_{k} u_{k}$ with their approximations (1.3), we get the F-A approximation of the function $v$ in the form $v=U+A+R$, where $A=\sum_{\mu=2}^{m} \alpha_{\mu} \sum_{k=n+1}^{+\infty} u_{k}(x) / k^{\mu}$ is a known function. The function $A$ we call the asymptotic correction. It is obvious that for the error of F-A approximation $R$ the estimate $R=O\left(1 / n^{m}\right), n \rightarrow+\infty$ is valid. Therefore, we can obtain a high accuracy of the result for small values of the parameter $n$, increasing the order of asymptotic approximation $m$.

The methods of asymptotic approximations for Fourier coefficients are well known in many classical cases [3].

Let the function $v=v(x)$ be smooth enough, except the point $x=\tau \in(0 ; 1)$ in which the function $v=v(x)$ and its derivatives can have the discontinuities of the first kind $\left(\Delta v_{k}=v^{(k)}(\tau+0)-v^{(k)}(\tau-0)\right)$. We find the asymptotic approximations (1.3) for the Fourier coefficients $c_{k}$ with the help of $2 m+2$ times integration by parts in the representation (1.2). Finally, we have the following F-A approximation for the function $v(x): v=U+A+R$, where $U$ is the partial sum of Fourier series (1.1);

$$
\begin{gathered}
A=2 \sum_{\mu=0}^{m} \varepsilon^{2 \mu+1}\left(v^{(2 \mu)}(1) \beta_{2 \mu+1}\left(\frac{x+1}{2}\right)-v^{(2 \mu)}(0) \beta_{2 \mu+1}\left(\frac{x}{2}\right)\right) \\
+\sum_{\mu=0}^{2 m+1}(-1)^{\mu+1} \varepsilon^{\mu+1} \mid \Delta v_{\mu}\left[\beta_{\mu+1}\left(\frac{x+\tau}{2}\right)-\beta_{\mu+1}\left(\frac{\tau-x}{2}\right)\right], \\
\left\{\begin{array}{l}
\beta_{2 \mu}(x)=(-1)^{\mu+1} \sum_{k=n+1}^{+\infty}\left(\frac{n+1}{k}\right)^{2 \mu} \cos 2 \pi k x, \\
\beta_{2 \mu+1}(x)=(-1)^{\mu+1} \sum_{k=n+1}^{+\infty}\left(\frac{n+1}{k}\right)^{2 \mu+1} \sin 2 \pi k x ;
\end{array}\right. \\
R=\varepsilon^{2 m+2} \int_{0}^{1} v^{(2 m+2)}(y)\left[\beta_{2 m+2}\left(\frac{x+y}{2}\right)-\beta_{2 m+2}\left(\frac{x-y}{2}\right)\right] d y
\end{gathered}
$$


and $\varepsilon=1 /(\pi(n+1)), \quad m=0,1, \ldots$

The F-A approximation consists of 1) the partial sum $U$ of Fourier series, 2) the asymptotic correction $A$ and 3 ) the explicit integral representation for the approximation error $R$. The asymptotic correction is lying in the ortogonal complement of the linear span of basic functions $\left\{u_{k}\right\}_{1}^{n}$ and is determined with the help of an additional analytic information about the approximate function $v=v(x): 1$ ) the values of its derivatives of even order at the endpoints, 2) the jumps of function $v(x)$ and its derivatives at the discontinuity point of the first kind $x=\tau$.

The functions $\beta_{k}(x)$ can be expressed with the help of the Lerch transcendent functions $\Phi(z, s, a)=\sum_{k=0}^{+\infty} z^{k} /(a+k)^{s}$ [2].

The F-A approximation is an asymptotic approximation with respect to the small parameter $\varepsilon=1 /(\pi(n+1))$. Here $n$ is the number of summands in the partial sum $U$ of the Fourier series. The error of approximation $R$ satisfies the estimate $R=o\left(\varepsilon^{2 m+2}\right), n \rightarrow+\infty$.

Therefore, the F-A approximation is applicable to the supported class of functions. But we can use more detailed information about analytical properties of function $v$. Let the function $v(x)$ be the solution of an ordinary differential equation. Then we obtain the derivatives of higher order of function $v(x)$ (which are necessary for the construction of asymptotic correction A) from the differential equation.

We give some examples that illustrates the application of F-A approximation for the solution of ordinary differential equations. We consider the problem of the Green function approximation, the solution of spectral problems and the solution of boundary problems for nonlinear equations. We construct the generalization of the classical Galerkin method for the solution of corresponding problems on the basis of F-A approximation.

\section{APPROXIMATION OF THE GREEN FUNCTION}

Let us regard the problem of Green's function $G=G(x, \tau)$ determination:

$$
\left\{\begin{array}{l}
G_{x x}^{\prime \prime}+\omega(x) G=\delta(x-\tau), \quad \tau \in(0,1), \\
\left.G\right|_{x=0}=\left.G\right|_{x=1}=0
\end{array}\right.
$$

where $\delta=\delta(x)$ is the Dirac $\delta$-function. We seek the Green function in the form $G=\varphi(x, \tau)+v(x, \tau)$, where

$$
\varphi(x, \tau)= \begin{cases}(\tau-1) x & , \quad 0<x \leq \tau \\ \tau(x-1) & , \quad \tau \leq x<1\end{cases}
$$

For the determination of function $v(x, \tau)$ we obtain the problem:

$$
v_{x x}^{\prime \prime}+\omega(x) v=-\omega(x) \varphi(x, \tau),\left.\quad v\right|_{x=0}=\left.v\right|_{x=1}=0 .
$$


We assume that the function $\omega=\omega(x)$ is smooth enough and we don't indicate the dependence of $v$ on the variable $\tau$, that is $v=v(x)$. We determine this function with the help of F-A approximation.

The function $v=v(x)$ is the solution of problem (2.3). Hence, all its derivatives of higher order, which are necessary for the construction of asymptotic correction $A$, can be expressed with $v$ and its derivative of the first order $v_{x}^{\prime}$ :

$$
v^{(k)}(x)=q_{k}(x)(\varphi+v)+p_{k}(x)\left(\varphi_{x}^{\prime}+v_{x}^{\prime}\right), \quad x \neq \tau, \quad k=2,3, \ldots,
$$

where $q_{k}$ and $p_{k}$ are defined with the recurrence relations:

$$
\left\{\begin{array}{l}
q_{k+1}(x)=q_{k}^{\prime}(x)-\omega(x) p_{k}(x), \quad p_{k+1}(x)=q_{k}(x)+p_{k}^{\prime}(x), \\
k=2,3, \ldots, \quad q_{2}(x)=-\omega(x), \quad p_{2}(x)=0 .
\end{array}\right.
$$

Finally we have the following F-A approximation of the Green function:

$$
G=W+R, \quad W=\varphi+U+A,
$$

where $U$ is the partial sum of the Fourier series. The asymptotic correction $A$ is following:

$$
\left\{\begin{array}{l}
A=s \Omega(x, 0)-z \Omega(x, 1)+\Omega(x, \tau) \\
s=G_{x}^{\prime}(0, \tau), \quad z=G_{x}^{\prime}(1, \tau) \\
\Omega(x, \tau)=\sum_{\mu=3}^{2 m+1}(-1)^{\mu+1} \varepsilon^{\mu+1} p_{\mu}(\tau)\left[\beta_{\mu+1}\left(\frac{x+\tau}{2}\right)-\beta_{\mu+1}\left(\frac{\tau-x}{2}\right)\right]
\end{array}\right.
$$

The error of the approximation is defined as

$$
\left\{\begin{array}{l}
R=R(x, \tau)=\varepsilon^{2 m+2} \int_{0}^{1} \Phi_{2 m+2}(t, \tau)\left[\beta_{2 m+2}\left(\frac{x+t}{2}\right)-\beta_{2 m+2}\left(\frac{x-t}{2}\right)\right] d t \\
\Phi_{2 m+2}(t, \tau)=q_{2 m+2}(t) G(t, \tau)+p_{2 m+2}(t) G_{t}^{\prime}(t, \tau), \quad \varepsilon=\frac{1}{\pi(n+1)} .
\end{array}\right.
$$

The F-A approximation (2.6) together with the error of approximation satisfy the boundary conditions of the problem (2.1). The F-A approximation gives us the possibility of a priori choice of parameters $n$ and $m$ values. This analysis is based on the investigation of the "velocity of decrease" for the sequences $\varepsilon^{\mu}\left|q_{\mu}(x)\right|$ and $\varepsilon^{\mu}\left|p_{\mu}(x)\right|, \mu=2,3, \ldots, 2 m+2$ (see Table $5)$. If the sequences decrease slowly, then it is necessary to extend $n$, since $\varepsilon=1 /(\pi(n+1))$. 
We must find the values of $s=G_{x}^{\prime}(0, \tau), z=G_{x}^{\prime}(1, \tau)$ and the Fourier coefficients $c_{1}, c_{2}, \ldots, c_{n}$ in the approximation (2.6). We obtain the system of $n+2$ equations for the determination of these parameters. The first $n$ equations are obtained by applying the ordinary Galerkin scheme, the last two equations are obtained by the differentiation of approximation (2.6) with respect to $x$ and substitution of $x=0$ and $x=1$. Therefore, we get some generalization of classical Galerkin method. Approximation (2.6), (2.7) has the following property. If the value of parameter $n$ is fixed and we increase the order of asymptotic approximation $m$, then the number of free parameters $c_{0}, \ldots, c_{n}, s$ and $z$ is not increased. In this case the approximation function $\Omega$ changes so that we obtain a more accurate result. The cause of this property is that we get all derivatives of higher order (which are necessary for the asymptotic correction $A$ ) from the given differential equation.

Example 1. Let $\omega(x)=a x+b$. Then the Green function $G(x, \tau)$ can be expressed with the help of the Airy functions.

Table 1. $\quad \tau=0.8, \quad a=0.8, \quad b=-0.5, \quad n=0$

\begin{tabular}{|c|l|l|l|l|c|}
\hline $\mathrm{x}$ & 0.2 & 0.6 & 0.8 & 0.9 & $\mathrm{~m}$ \\
\hline $\mathrm{G}$ & $-3.93949 \cdot 10^{-2}$ & -0.1197029 & -0.1601757 & $-8.017065 \cdot 10^{-2}$ & - \\
\hline $\mathrm{W}$ & $-3.93937 \cdot 10^{-2}$ & -0.1197009 & -0.1601731 & $-8.016920 \cdot 10^{-2}$ & 3 \\
\hline $\mathrm{W}$ & $-3.93959 \cdot 10^{-2}$ & -0.1197043 & -0.1601751 & $-8.017029 \cdot 10^{-2}$ & 6 \\
\hline $\mathrm{W}$ & $-3.93959 \cdot 10^{-2}$ & -0.1197098 & -0.1601751 & $-8.017028 \cdot 10^{-2}$ & 12 \\
\hline
\end{tabular}

Table 2. $\quad \tau=0.8, \quad a=2.1, \quad b=1.0, \quad n=0$

\begin{tabular}{|c|l|l|l|l|c|}
\hline $\mathrm{x}$ & 0.2 & 0.6 & 0.8 & 0.9 & $\mathrm{~m}$ \\
\hline $\mathrm{G}$ & $-5.629675 \cdot 10^{-2}$ & -0.1540872 & -0.1849851 & $-9.382869 \cdot 10^{-2}$ & - \\
\hline $\mathrm{W}$ & $-5.881873 \cdot 10^{-2}$ & -0.1582060 & -0.1875406 & $-9.517408 \cdot 10^{-2}$ & 3 \\
\hline $\mathrm{W}$ & $-5.514153 \cdot 10^{-2}$ & -0.1521920 & -0.1838060 & $-9.320741 \cdot 10^{-2}$ & 6 \\
\hline
\end{tabular}

Table 3. $\quad \tau=0.8, \quad a=2.8, \quad b=1.0$ and different $\mathrm{n}, \mathrm{m}$

\begin{tabular}{|c|l|l|l|l|c|}
\hline $\mathrm{x}$ & 0.2 & 0.6 & 0.8 & 0.9 & $\mathrm{n} / \mathrm{m}$ \\
\hline $\mathrm{G}$ & $-6.008587 \cdot 10^{-2}$ & -0.1623818 & -0.1911455 & $-9.725691 \cdot 10^{-2}$ & - \\
\hline $\mathrm{W}$ & $-5.554543 \cdot 10^{-2}$ & -0.1548930 & -0.1864750 & $-9.479377 \cdot 10^{-2}$ & $0 / 6$ \\
\hline $\mathrm{W}$ & $-6.008588 \cdot 10^{-2}$ & -0.1623818 & -0.1911454 & $-9.725698 \cdot 10^{-2}$ & $2 / 7$ \\
\hline $\mathrm{W}$ & $-6.008587 \cdot 10^{-2}$ & -0.1623818 & -0.1911454 & $-9.725698 \cdot 10^{-2}$ & $5 / 3$ \\
\hline $\mathrm{W}$ & $-6.008587 \cdot 10^{-2}$ & -0.1623818 & -0.1911454 & $-9.725698 \cdot 10^{-2}$ & $1 / 12$ \\
\hline
\end{tabular}

Table 4. $\quad \tau=0.3, \quad a=16, \quad b=8$ and different $\mathrm{n}, \mathrm{m}$

\begin{tabular}{|c|l|l|l|l|c|}
\hline $\mathrm{x}$ & 0.2 & 0.3 & 0.6 & 0.8 & $\mathrm{n} / \mathrm{m}$ \\
\hline $\mathrm{G}$ & 0.0292836 & $3.989039 \cdot 10^{-2}$ & 0.2726635 & 0.2202688 & - \\
\hline $\mathrm{W}$ & 0.0292837 & $3.989062 \cdot 10^{-2}$ & 0.2726636 & 0.2202691 & $3 / 12$ \\
\hline $\mathrm{W}$ & 0.0292838 & $3.989069 \cdot 10^{-2}$ & 0.2726637 & 0.2202689 & $5 / 6$ \\
\hline
\end{tabular}


If absolute values of $a$ and $b$ are small, we obtain a high accuracy of the approximation even with $n=0$ (Table 1). When the absolute values of $a$ and $b$ are increased, the accuracy of the approximation with respect to $n=0$ falls (Table 2). It is necessary in this case to increase the value of $n$ (Tables 3 and 4 ). We choose values for the parameters $n, m$ and make a priori estimate of accuracy with the help of numerical analysis for the "velocity of decrease" of the sequences $\varepsilon^{\mu} q_{\mu}(x)$ and $\varepsilon^{\mu} p_{\mu}(x)$.

In Table 5 are presented the values of members of the sequence are presented $\varepsilon^{\mu} p_{\mu}(x)$ for different $n$.

Table 5. $\quad \varepsilon^{\mu} p_{\mu}(x), \quad x=\tau, \quad a=2.8, \quad b=1, \quad \tau=0.8$

\begin{tabular}{|c|l|l|l|l|}
\hline$\mu$ & 3 & 4 & 14 & \\
\hline$\varepsilon^{\mu} p_{\mu}$ & -0.104495 & $-5.748949 \cdot 10^{-2}$ & $-5.905703 \cdot 10^{-2}$ & $n=0$ \\
\hline \hline$\mu$ & 3 & 8 & 25 & \\
\hline$\varepsilon^{\mu} p_{\mu}$ & $-1.306187 \cdot 10^{-2}$ & $-1.452079 \cdot 10^{-4}$ & $-1.663331 \cdot 10^{-8}$ & $n=1$ \\
\hline \hline$\mu$ & 3 & 4 & 14 & \\
\hline$\varepsilon^{\mu} p_{\mu}$ & $-3.870184 \cdot 10^{-3}$ & $-7.097470 \cdot 10^{-4}$ & $-1.234736 \cdot 10^{-8}$ & $n=2$ \\
\hline \hline$\mu$ & 3 & 4 & 7 & \\
\hline$\varepsilon^{\mu} p_{\mu}$ & $-4.837731 \cdot 10^{-4}$ & $-4.435919 \cdot 10^{-5}$ & $5.249955 \cdot 10^{-8}$ & $n=5$ \\
\hline
\end{tabular}

\section{SOLUTION OF SPECTRAL PROBLEMS}

We consider the eigenvalue problem:

$$
v^{\prime \prime}+\omega(x, \lambda) v(x)=0, \quad x \in(0 ; 1), \quad v(0)=v(1)=0
$$

If we apply the F-A approximation for the solution of equation (3.1), then we obtain the representation: $v=U+A+R$, where $A(x, \lambda)=s \omega(x, \lambda)-$ $z g(x, \lambda)$ and

$$
\begin{gathered}
\omega(x, \lambda)=-2 \sum_{\mu=2}^{m} \varepsilon^{2 \mu+1} p_{2 \mu}(0, \lambda) \beta_{2 \mu+1}\left(\frac{x}{2}\right), \quad s=v^{\prime}(0), \\
g(x, \lambda)=-2 \sum_{\mu=2}^{m} \varepsilon^{2 \mu+1} p_{2 \mu}(1, \lambda) \beta_{2 \mu+1}\left(\frac{x+1}{2}\right), \quad z=v^{\prime}(1), \\
R(x, \lambda)=\varepsilon^{2 m+2} \int_{0}^{1} \Phi_{2 m+2}(y, \lambda)\left[\beta_{2 m+2}\left(\frac{x+y}{2}\right)-\beta_{2 m+2}\left(\frac{x-y}{2}\right)\right] d y \\
\Phi_{2 m+2}(y, \lambda)=q_{2 m+2}(y, \lambda) v(y)+p_{2 m+2}(y, \lambda) v^{\prime}(y), \quad \varepsilon=\frac{1}{\pi(n+1)}
\end{gathered}
$$


The sequences $\left\{q_{k}\right\}$ and $\left\{p_{k}\right\}$ can be determined with the help of recurrence relations:

$$
\left\{\begin{array}{l}
q_{2}=-\omega(x, \lambda), \quad p_{2}=0 \\
q_{k+1}(x, \lambda)=\frac{\partial}{\partial x} q_{k}(x, \lambda)-\omega(x, \lambda) p_{k}(x, \lambda) \\
p_{k+1}(x, \lambda)=q_{k}(x, \lambda)+\frac{\partial}{\partial x} p_{k}(x, \lambda)
\end{array}\right.
$$

Again, it is possible to select apriori the values of the parameters $n$ and $m$ when we analyze the "velocity of decrease" for the sequences $\varepsilon^{\mu} q_{\mu}(x, \lambda)$ and $\varepsilon^{\mu} p_{\mu}(x, \lambda)$. The optimal values of parameters $n$ and $m$ depend essentially on the domain of variable $\lambda$ in which we seek the eigenvalues.

The details of constructions are similar to the previous example. Finally, we obtain some generalization of the Galerkin method for the determination of eigenvalues and eigenfunctions.

Example 2. Let $\omega(x, \lambda)=a x+\lambda$, where $a \in \mathbf{R}$. In table 6 we show the first two eigenvalues for the parameter $a=5, a=-7, a=-17$ and their approximations with the computational Galerkin method for $n=2$. In the last four rows we show the results of $\mathrm{F}$-A approximation for $n=2$ and different values of $m$.

Table 6 .

\begin{tabular}{|c|c|c|c|c|c|c|}
\hline \multicolumn{2}{|c|}{$a=5$} & \multicolumn{2}{c|}{$a=-7$} & \multicolumn{2}{c|}{$a=-17$} & \multirow{2}{*}{} \\
\cline { 1 - 5 }$\lambda_{1}$ & $\lambda_{2}$ & $\lambda_{1}$ & $\lambda_{2}$ & $\lambda_{1}$ & $\lambda_{2}$ & \\
\hline 7.342186 & 36.98655 & 13.31589 & 42.99436 & 18.05433 & 48.07121 & exact \\
\hline 7.342234 & 37.00579 & 13.31601 & 43.03202 & 18.05623 & 48.29179 & Galer. \\
\hline 7.342190 & 36.99809 & 13.31591 & 43.01693 & 18.05470 & 48.20333 & $m=3$ \\
\hline 7.342186 & 36.99124 & 13.31589 & 42.99948 & 18.05429 & 48.08890 & $m=5$ \\
\hline 7.342186 & 36.98804 & 13.31589 & 42.99702 & 18.05432 & 48.06232 & $m=7$ \\
\hline 7.342186 & 36.98694 & 13.31589 & 42.99484 & 18.05433 & 48.06008 & $m=9$ \\
\hline
\end{tabular}

Example 3. Let $\omega(x, \lambda)=\lambda x$. In Table 7 we show the first eigenvalue $\lambda_{1}$, its approximation with Galerkin method for $n=2$ and the results of F-A approximation for $n=2$ and different values of $m$.

Table 7 .

\begin{tabular}{|c|c|}
\hline$\lambda_{1}$ & \\
\hline 18.95626 & exact \\
\hline 18.96146 & Galerkin \\
\hline 18.95950 & $m=2$ \\
\hline 18.95643 & $m=4$ \\
\hline 18.95615 & $m=6$ \\
\hline 18.95627 & $m=8$ \\
\hline
\end{tabular}

Table 8 .

\begin{tabular}{|l|l|c|}
\hline$\lambda_{1}$ & $\lambda_{2}$ & \\
\hline 6.4075 & 28.437 & Collatz \\
\hline 6.414262 & 29.34737 & Galerkin \\
\hline 6.407765 & 28.86608 & $m=2$ \\
\hline 6.407703 & 28.61269 & $m=4$ \\
\hline 6.407703 & 28.43717 & $m=10$ \\
\hline 6.407703 & 28.43717 & $m=18$ \\
\hline
\end{tabular}

The proposed algorithm can be easily generalized for the case when the function $\omega(x, \lambda)$ has a discontinuity of the first kind with respect $x$. 
Example 4. Let $\omega(x, \lambda)=\lambda \omega(x)$, where $\omega(x)=\left\{\begin{array}{ll}2, & 0 \leq x<1 / 2 \\ 1, & 1 / 2 \leq x<1\end{array}\right.$. We show in table 8 the first two eigenvalue $\lambda_{1}$ and $\lambda_{2}$ which are given by Collatz [1]. In the second row we present their Galerkin approximations for $n=2$. In the next rows the results for $n=2$ and different $m$ are given.

The numerical experiments show that, if the approximation is possible for small $n$, then we can obtain a high accuracy of results by increasing $m$.

This fact allows us to apply the F-A approximation to the analysis of nonlinear equations.

\section{SOLUTION OF NONLINEAR EQUATIONS}

Let us consider the following boundary problem:

$$
y^{(2)}=\omega y+t y^{2}+f_{0}, \quad x \in(0 ; 1), \quad y^{\prime}(0)=z, \quad y^{\prime}(1)=s,
$$

where $\omega, t, f_{0}, z, s$ are parameters. We seek the solution of equation (4.1) in the form

$$
y(x)=H(x)+v(x), \quad H(x)=z x+(s-z) x^{2} / 2 .
$$

We determine the new function $v(x)$ with the help of F-A cosine approximation. Such an approximation can be constructed in the same way as the F-A sine approximation. We consider the parameters $n=1$ and $m=3$. Then we obtain the following F-A approximation for the solution of equation (4.1):

$$
\begin{gathered}
y(x)=H(x)+c_{0}+c_{1} \cos \pi x+A(x)+R(x), \\
A(x)=2 \sum_{\mu=2}^{3} \varepsilon^{2 \mu}\left(\varphi_{2 \mu-1}(\sigma, s) \beta_{2 \mu}\left(\frac{x+1}{2}\right)-\varphi_{2 \mu-1}(\kappa, z) \beta_{2 \mu}\left(\frac{x}{2}\right)\right), \\
R(x)=\varepsilon^{7} \int_{0}^{1} \varphi_{7}\left(y(\tau), y^{\prime}(\tau)\right)\left[\beta_{7}\left(\frac{x+\tau}{2}\right)+\beta_{7}\left(\frac{\tau-x}{2}\right)\right] d \tau,
\end{gathered}
$$

here

$$
\begin{aligned}
& \left\{\begin{array}{l}
\varphi_{3}\left(y, y^{\prime}\right)=\omega y^{\prime}+2 t y y^{\prime} \\
\varphi_{5}\left(y, y^{\prime}\right)=\omega^{2} y^{\prime}+10 \omega t y y^{\prime}+10 t^{2} y^{2} y^{\prime}+6 t f_{0} y^{\prime} \\
\varphi_{7}\left(y, y^{\prime}\right)=\omega^{3} y^{\prime}+42 \omega^{2} t y y^{\prime}+120 \omega t^{2} y^{2} y^{\prime} \\
+80 t^{3} y^{3} y^{\prime}+36 \omega t f_{0} y^{\prime}+20 t^{2} y^{\prime 3}
\end{array}\right. \\
& \varepsilon=1 /(2 \pi), \quad \kappa=y(0), \quad \sigma=y(1) .
\end{aligned}
$$


It should be noted that the functions $\varphi_{k}\left(y, y^{\prime}\right)=y^{(k)}$ are obtained with the help of a successive differentiation of the equation (4.1).

The integral representation (4.5) allows us to determine a priori the domain of variation for the basic parameters of our problem: $\omega, t, z, s, f_{0}$. In this domain we can disregard the error of approximation $R(x)$ and regard the values of function $A(x)$ as small. The absolute values of basic parameters must not exceed "substantially" the values of the selected parameters $n=1$ and $m=3$.

We have a system of four equations for the determination of the Fourier coefficients $c_{0}, c_{1}$ and the values of variables $\kappa=y(0), \sigma=y(1)$. We obtain the first two equations with the help of the Galerkin method:

$$
\left\{\begin{array}{ccc}
p_{0}^{2}+p_{1}^{2} & = & g_{0} \\
\left(p_{0}+\alpha\right) p_{1} & = & g_{1}
\end{array}\right.
$$

where

$$
\begin{gathered}
c_{0}=\frac{\pi^{2} p_{0}-\omega}{2 t}-\frac{1}{6}(s+2 z), \quad c_{1}=\frac{\pi^{2} p_{1}}{\sqrt{2} t}+\frac{2}{\pi^{2}}(z+s), \\
g_{0}=\frac{\omega^{2}}{\pi^{4}}-\frac{4 t}{\pi^{4}}\left(z-s+f_{0}\right)-\frac{8 t^{2}}{\pi^{8}}\left[\frac{\pi^{4}-90}{90}\left(z^{2}+s^{2}\right)+\frac{7 \pi^{4}-720}{360} z s\right]-\frac{4 t^{2}}{\pi^{4}} \gamma_{0}, \\
\alpha=1+\frac{t}{2 \pi^{4}}(s-z)+\frac{2 t}{\pi^{2}}\left(A_{1}+R_{1}\right), \\
g_{1}=-\frac{2{ }^{2} t}{\pi^{4}}(s+z)-\frac{\sqrt{2} t^{2}}{\pi^{8}} \frac{4 \pi^{2}-39}{3}\left(z^{2}-s^{2}\right)-\frac{2 \sqrt{2} t^{2}}{\pi^{4}} \gamma_{1}, \\
\gamma_{0}^{1}\left[2 H(x)(A(x)+R(x))+(A(x)+R(x))^{2}\right] d x, \\
\gamma_{1}=\int_{0}^{1}\left[2 H(x)(A(x)+R(x))+(A(x)+R(x))^{2}\right] \cos \pi x d x+\frac{2}{\pi^{2}}(z+s)\left(A_{1}+R_{1}\right), \\
A_{1}=\sum_{\mu=2}(-1)^{\mu+1} \varepsilon^{2 \mu}\left(\varphi_{2 \mu-1}(\sigma, s)-\varphi_{2 \mu-1}(\kappa, z)\right),
\end{gathered}
$$


We get another two equations when we substitute the values $x=0$ and $x=1$ into the approximation (4.3)

$$
\left\{\begin{array}{l}
\kappa=c_{0}+c_{1}+A(0)+R(0) \\
\sigma=\frac{1}{2}(s+z)+c_{0}-c_{1}+A(1)+R(1) .
\end{array}\right.
$$

Let us carry out a qualitative analysis. We introduce the function

$$
\Psi\left(g_{0}\right)=\frac{\alpha^{2} g_{0}}{32 h}(2+h)^{3}, \quad h=1+\sqrt{1+\frac{8 g_{0}}{\alpha^{2}}},
$$

which monotonically increases for $g_{0} \geq 0$. Then

1) for $\Psi\left(g_{0}\right)<g_{1}^{2}$ the system has no solution;

2) for $\Psi\left(g_{0}\right)=g_{1}^{2}$ the system has one solution

$$
p_{0}=\frac{2 g_{0}}{\alpha h}, \quad p_{1}=\frac{g_{1}}{\alpha+p_{0}}
$$

3) for $\Psi\left(g_{0}\right)>g_{1}^{2}$ and

$$
\Theta\left(g_{0}\right)=\frac{2\left(g_{0}-\alpha^{2}\right)^{3} h}{(2+h) \alpha^{2}}<g_{1}^{2}
$$

the system has two solutions, but for $\Theta\left(g_{0}\right)=g_{1}^{2}$ third solution emerge:

$$
p_{0}=-\frac{\alpha h}{4}, \quad p_{1}=-\frac{g_{1} \alpha(2+h)}{2\left(g_{0}-\alpha^{2}\right)} ;
$$

4) for $\Theta\left(g_{0}\right)>g_{1}^{2}$ the system has four solutions.

When we further increase the value of the parameter $g_{0}$, we leave the admissible domain of approximation (by $n=1$ and $m=3$ ).

Thus, the equations $\Psi\left(g_{0}\right)=g_{1}^{2}, \Theta\left(g_{0}\right)=g_{1}^{2}$ give the first two surfaces of bifurcations in the pentadimensional space of parameters $\omega, t, z, s$ and $f_{0}$. If $t=z=s=f_{0}=0$, then we get from these equations that $\omega=0$ and $\omega=\pi^{2}$. They are the first two eigenvalues of corresponding linear spectral problem.

We also can obtain an approximate solution for the problem (4.1), if we apply a proper iterative process. For example, if we don't take into account the errors $R(x)$ and $R_{1}$ and suppose that $\kappa=y(0)=0, \sigma=y(1)=0$, then we can obtain the solution of the system (4.9). Next, we iteratively determine the values of parameters $\kappa$ and $\sigma$ from the system (4.11) and solve again the system (4.9). So, we construct the iterative loop. When we determine the solution $y(x)$, we can obtain apriori the error estimate from the representation $(4.5)$. 


\section{CONCLUSION}

The main idea of the Fourier-asymptotic (F-A) approximation is that starting with some index $n$ we substitute the Fourier coefficients with their asymptotic $(n \rightarrow+\infty)$ approximations.

With the help of F-A approximation we get some generalization of the classical Galerkin method for the solution of boundary problem for ordinary differential equations. The main merit of this modification is the possibility apriori selection of the number $n$ of basic functions, which guarantee the small error. Moreover, we can have both the apriori and the a posteriori estimates of the approximation error. We can clarify the domain of applicability for the F-A approximation. The numerical examples show that the addition of asymptotic correction allows us to obtain a high accuracy of results for small $n$. This allows us to apply the F-A approximation for solving nonlinear equations. In this case the F-A approximation allows us to carry out an approximate analysis of a decidability for the nonlinear boundary problem, to obtain the analytic expressions for the surface of bifurcation and to construct the approximate solution.

We can obtain the Fourier-asymptotic approximation for different types of Fourier series, one dimensional or multidimensional. We need only to find the asymptotic approximations for the corresponding Fourier coefficients. Analogous approximations can be constructed for the integral Fourier transform.

\section{REFERENCES}

[1] L. Collatz, Eigenwertaufgaben mit technischen Anwendungen, Acad. Verlagsgesellschaft Geest and Portig K.-G., Leipzig, 1963.

[2] Y. Luke, Mathematical functions and their approximations, Acad. Press, New York; London, 1975.

[3] E. I. Riekstiņš, Asymptotic expansion of integrals, Zinātne, Rīga, 1974, Vol. $1-3$ (in Russian). 